Ilya Kister, MD

Eric Chamot, MD, PhD

Amber R. Salter, MPH

Gary R. Cutter, PhD

Tamar E. Bacon, BA

Joseph Herbert, MD

Correspondence to

Dr. Kister:

ilya.kister@gmail.com

\section{Disability in multiple sclerosis}

\author{
A reference for patients and clinicians
}

\section{ABSTRACT}

Objective: To create a reference table of disability outcomes in multiple sclerosis (MS) that would enable patients to rank their disability relative to others' with similar disease duration and to develop a cost-effective research tool for comparing MS severity across patient populations and time periods.

Methods: The North American Research Committee on Multiple Sclerosis (NARCOMS) Registry collects disability data from patients with MS on a validated, 9-point Patient-Determined Disease Steps (PDDS) scale. We compiled the Disability Expectancy Table, which displays cumulative frequencies of PDDS scores for each year of disease duration, from 0 to 45 years. We also tabulated disease duration-adjusted mean ranks of PDDS scores, referred to as Patient-derived MS Severity Scores (P-MSSS).

Results: The cohort consisted of 27,918 NARCOMS enrollees, $72.7 \%$ of whom were female and $90.1 \%$ of whom were white. Mean age at symptom onset was $30.1 \pm 10.1$ years, and age at enrollment was $47.1 \pm 11.0$ years. The Disability Expectancy Table and P-MSSS afford a detailed overview of disability outcomes in a large MS cohort over a 45-year period. In the first year of disease, $15 \%$ of patients reported need of ambulatory aid, and $4 \%$ needed bilateral assistance or worse; after 45 years of disease, $76 \%$ of patients required ambulatory aid, and $52 \%$ bilateral assistance or worse. Proportion of patients who reported minimal or no interference in daily activities (PDDS $\leq 1$ ) declined from $63 \%$ in the first year to $8 \%$ after 45 years of disease.

Conclusion: The Disability Expectancy Table allows individual patients to determine how their disability ranks relative to NARCOMS enrollees with the same disease duration. P-MSSS may be used to compare disability across patient populations and to track disease progression in patient cohorts. P-MSSS does not require a formal neurologic examination and may therefore find wide applicability as a practical and cost-effective outcome measure in epidemiologic studies. Neurology ${ }^{\circledast}$ 2013;80:1018-1024

\section{GLOSSARY}

DET = Disability Expectancy Table; EDSS = Expanded Disability Status Scale; GAMES = Genetic Analysis of Multiple Sclerosis in Europeans; MS = multiple sclerosis; MSSS = Multiple Sclerosis Severity Score; NARCOMS = North American Research Committee on Multiple Sclerosis; NHIS-D = National Health Interview Survey on Disability; P-MSSS = Patientderived MS Severity Score; PDDS = Patient-Determined Disease Steps.

The natural history of multiple sclerosis (MS) has been charted extensively, ${ }^{1,2}$ but there are no easyto-use references of disability outcomes that would enable individual patients to determine how their disability compares to others with similar disease duration. Representation of disability outcomes as a table of mean ranks of Expanded Disability Status Scale (EDSS) scores in patients with comparable disease duration, the Global Multiple Sclerosis Severity Score (MSSS) Table, ${ }^{3}$ has proven fruitful for epidemiologic studies, ${ }^{4-7}$ but as a reference for patients and clinicians, MSSS's utility is limited by the requirement for trained clinicians' assessment of EDSS score and lack of a simple, intuitive interpretation.

The aim of this work is to develop a patient- and clinician-friendly reference table of disability outcomes in MS, the Disability Expectancy Table (DET). The reference cohort for DET derives

From the Department of Neurology (I.K., T.E.B., J.H.), NYU-Multiple Sclerosis Care Center, NYU School of Medicine, New York, NY; and the Departments of Epidemiology (E.C.) and Biostatistics (A.R.S., G.R.C.), University of Alabama at Birmingham School of Public Health, Birmingham. Go to Neurology.org for full disclosures. Funding information and disclosures deemed relevant by the authors, if any, are provided at the end of the article. 
from the North American Research Committee on Multiple Sclerosis (NARCOMS) Registry, a nearly 36,000-patient-strong volunteer registry that collects disability data on a validated Patient-Determined Disease Steps (PDDS) scale (appendix). ${ }^{8} 9$ DET displays maximum ranks (cumulative frequencies) of PDDS scores for disease durations of $0-45$ years. DET allows easy determination of how a patient's disability compares to others with same disease duration.

To make our work more relevant for clinical researchers, we tabulate mean ranks of PDDS scores for each year of disease. The mean ranks, Patient-determined Multiple Sclerosis Severity Scores (P-MSSS), stand in the same relation to PDDS as MSSS does to EDSS. P-MSSS does not require clinician input and could become a practical and cost-effective alternative outcome measure for epidemiologic research.

METHODS The NARCOMS Registry has enrolled over 35,000 patients with self-reported diagnosis of MS from 1996 to present. ${ }^{8}$ Participants complete the institutional review board-approved enrollment form offered online and on paper, which includes information on demographics, disease history, medication history, and disability as assessed with PDDS scale. ${ }^{9}$ Key clinical elements of the registry have been validated in a random sample of participant ${ }^{10-12}$ or by comparison to standardized scales. Only registrants with disease duration of $0-45$ years at the time of initial PDDS were included; the number of patients with disease duration of $>45$ years was insufficient for statistical analyses.

Maximum ranks of PDDS scores (0-8) were calculated for each subgroup of NARCOMS enrollees with same disease duration. Maximum ranks were then divided by the number of patients within their disease duration strata to obtain the proportion (cumulative frequency) of patients whose disability is equal to or less than respective PDDS scores. We present cumulative frequencies of PDDS scores for years $0-45$ from symptom onset in the DET.

P-MSSS were derived using the same frequency-ranking algorithm as for MSSS. ${ }^{3}$ In brief, within each year of disease duration, PDDS scores were ranked, and for each possible PDDS value (0-8), the average of the lowest and highest ranks was calculated. P-MSSS is the mean rank, expressed as deciles, of PDDS scores of patients with similar disease duration. To reduce stochastic fluctuations in estimation of mean ranks, each year was analyzed with 2 preceding and 2 subsequent years of disease duration (e.g., year 5 from symptom onset includes patients with disease duration of 3, 4, 5, 6, and 7 years). P-MSSS distributions during the first 45 years of disease are shown in the P-MSSS Table.

RESULTS As of June 2011, the NARCOMS Registry contained records on 35,930 participants from the United States. Because of missing age at enrollment, age at symptom onset, PDDS, or disease duration $>45$ years, 8,012 NARCOMS enrollees were excluded from the study. The reference cohort consisted of 27,918 NARCOMS registrants, each contributing only their enrollment PDDS score to our analysis. Demographic and disease-related characteristics of this cohort are shown in the table.

The DET comprises 46 multicolored stripes. Each stripe represents a year from symptom onset, with year 0 at the top of the y-axis and year 45 at the bottom (figure 1). Stripes are divided into 9 segments corresponding to PDDS grades 0-8. PDDS segments are color-coded as explained in figure 1 . The outer boundary of PDDS segments on the $\mathrm{x}$-axis corresponds to cumulative frequency of patients with disability score equal to or lower than the respective PDDS scores. Cumulative frequencies, in percent, are shown for all PDDS at the right of the corresponding segment (except for PDDS $=8$ segments which, by definition, have cumulative frequency of $100 \%$ ). Use of the DET is illustrated in the legend to figure 1.

As can be seen from DET, the proportion of patients who reported being asymptomatic, or having minimal disease interference with daily function (PDDS of 0 and 1), falls rapidly from $63 \%$ in year 0 (outer boundary of peach-colored PDSS = 1 segment in the top stripe) to $32 \%$ by the end of the first decade, and continues to decline, at a slower pace, to $8 \%$ after 45 years of disease. Similar trends were seen for the other mild-to-moderate PDDS scores. The proportion of patients who reported being able to walk without any ambulatory assistanceouter boundary of green-colored PDDS $=3$ segment in figure 1 - decreased from $85 \%$ in year 0 to $60 \%$ at the end of the first decade, to $24 \%$ after 45 years. The proportion of those who recorded need for at least bilateral assistance to walk increased from $5 \%$ at year 0 to $13 \%$ at year 10 to $52 \%$ at year 45 .

\begin{tabular}{|c|c|c|}
\hline \multirow{2}{*}{\multicolumn{2}{|c|}{$\begin{array}{l}\text { Table Demographic and disease-r } \\
\text { characteristics of the NAR } \\
\text { at enrollment }\end{array}$}} & $\begin{array}{l}\text { Ited } \\
\text { MS cohort }\end{array}$ \\
\hline & & Values \\
\hline \multicolumn{2}{|c|}{ No. of patients } & 27,918 \\
\hline \multicolumn{2}{|c|}{$\%$ Female } & 72.7 \\
\hline \multicolumn{3}{|c|}{ Race, \% } \\
\hline \multicolumn{2}{|c|}{ White } & 90.1 \\
\hline \multicolumn{2}{|c|}{ African American } & 4.4 \\
\hline \multicolumn{2}{|c|}{ Hispanic } & 2.3 \\
\hline \multicolumn{2}{|c|}{ Other } & 3.2 \\
\hline \multicolumn{2}{|c|}{ Age at symptom onset, $y$, mean (SD) } & $30.1(10.1)$ \\
\hline \multicolumn{2}{|c|}{ Age at diagnosis, $y$, mean (SD) } & $37.3(9.9)$ \\
\hline \multicolumn{2}{|c|}{ Age at enrollment, y, mean (SD) } & 47.1 (11.0) \\
\hline \multicolumn{2}{|c|}{ Employed, \% } & 43.1 \\
\hline \multicolumn{2}{|c|}{ Taking immunomodulatory therapies, $\%^{\mathrm{a}}$} & 52.0 \\
\hline
\end{tabular}

Abbreviation: NARCOMS $=$ North American Research Committee on Multiple Sclerosis.

a Out of 11,790 respondents who answered the question. 


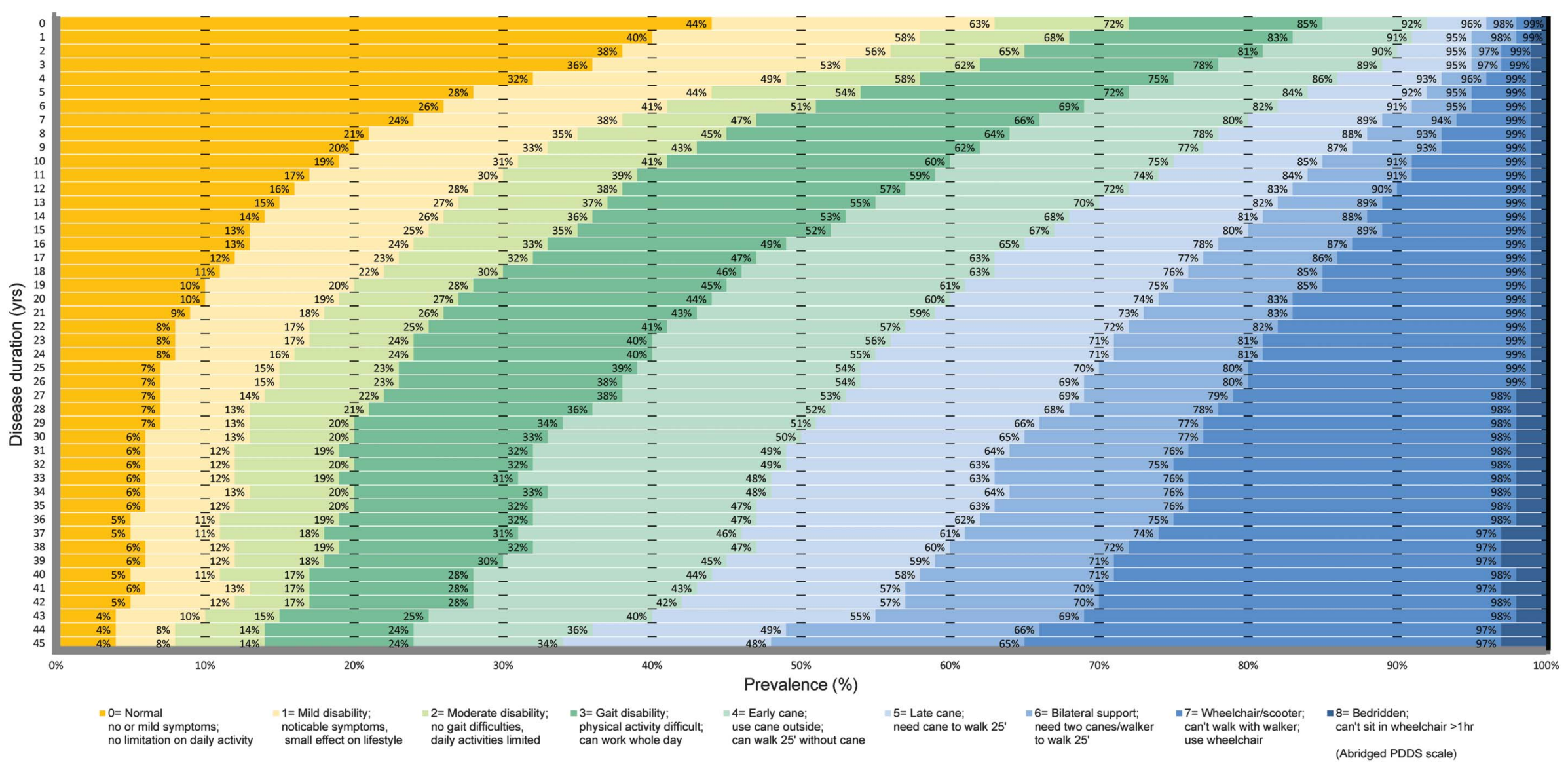

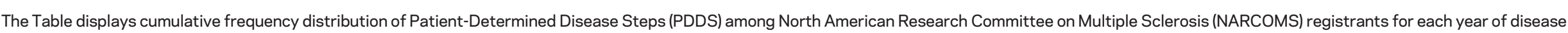

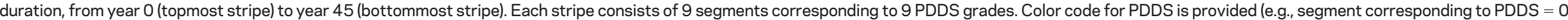

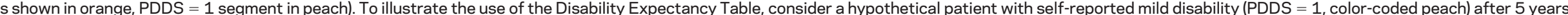

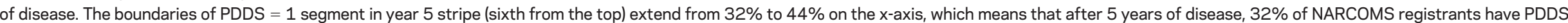

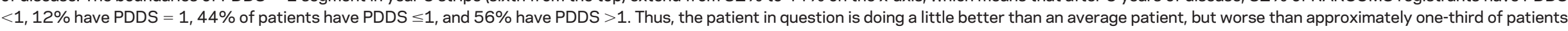

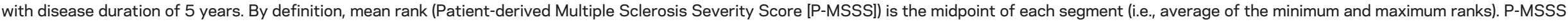
data are shown in figure 2 
Figure 2 Patient-derived Multiple Sclerosis Severity Score Table

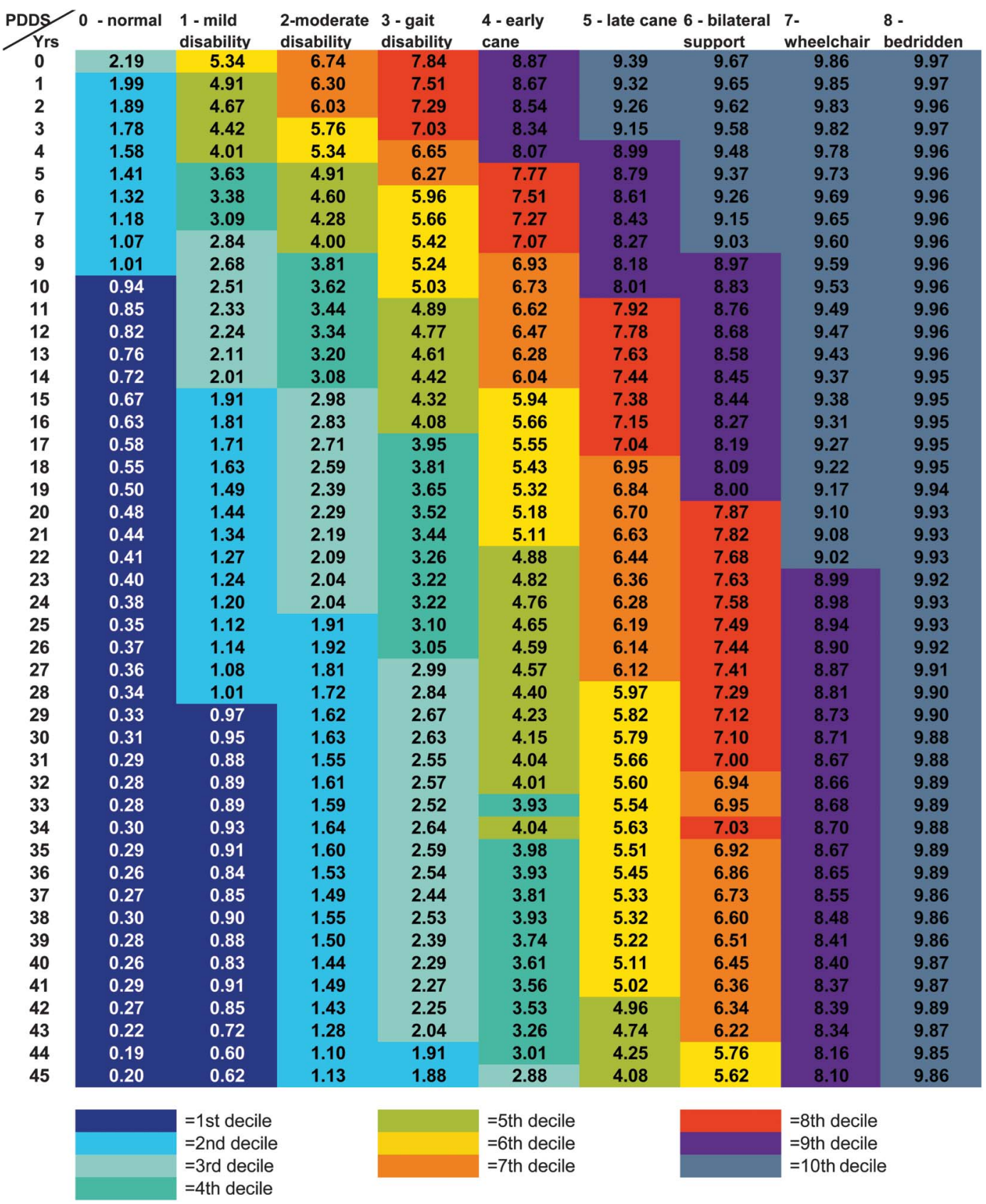

The format of this Table follows that of Global Multiple Sclerosis Severity Score (MSSS) Table ${ }^{3}$ with Patient-Determined Disease Steps (PDDS) on the $x$-axis and years of disease duration on the $y$-axis. To maintain consistency with MSSS, the Patient-derived MS Severity Scores (P-MSSS) values are reported in deciles, rather than percent. A different color is used for each decile of P-MSSS; color code is provided at the bottom of the Table.

The P-MSSS Table (figure 2) displays disease duration-adjusted mean ranks of PDDS. Its format parallels that of the Global MSSS Table ${ }^{3}$ to highlight the similarities between the two. The P-MSSS Table is comprised of 9 columns corresponding to PDDS grades $0-8$, respectively, and 46 rows corresponding to years 0 to 45 from symptom onset. P-MSSS for every PDDS/year of disease is shown inside the box at the intersection of the 2 coordinates. The P-MSSS Table is color-coded using the same color scheme as
MSSS, ${ }^{3}$ with each decile of P-MSSS corresponding to a specific color (legend to figure 2).

DISCUSSION The DET, based on nearly 28,000 individual patient registrations, affords a uniquely detailed representation of patient-rated disability outcomes in MS during the first 45 years of disease. The DET demonstrates that a considerable minority of patients perceive their disease as exerting a significant toll on their dayto-day functioning as early as the first few years after 
onset. The proportion of patients with PDDS $=3$ ("MS does interfere with my activities, especially my walking; I can work a full day...”) or worse reaches $50 \%$ after 15 years of disease and $75 \%$ after 45 years. If PDDS $=3$ is taken as an approximate disability benchmark beyond which most patients would find it difficult to maintain full-time employment, then it is understandable why only $43 \%$ of the NARCOMS cohort, whose mean disease duration was 17 years, were fully employed. Similar low rates of employment were documented in a recent National Multiple Sclerosis Society survey ${ }^{13}$ and in the New York State Multiple Sclerosis Consortium. ${ }^{14}$ On the positive side, approximately 1 in 4 patients rated their disability as being compatible with full-time employment (PDSS $\leq 3) 45$ years after disease onset. The latter estimate should be viewed as an upper bound given that the oldest and most disabled patients are likely underrepresented in the NARCOMS registry.

Comparison of mobility device use in NARCOMS and the general population further underscores the magnitude of MS impact. The National Health Interview Survey on Disability (NHIS-D) ${ }^{15}$ recorded that $1.5 \%$ of respondents aged 18-64 years in the general population used a mobility device, while among NARCOMS enrollees, the proportion of mobility device users in the same age group was $45 \%$, a 31-fold difference. In the 64 or older group, $14 \%$ of NHIS-D respondents used a mobility device, compared to $81 \%$ in NARCOMS, a 6-fold difference (data not shown).

The DET is based on a volunteer registry, and it is instructive to compare it with population-based natural history studies of MS (with the proviso that a comparison that relies on similar, but nonidentical scalesEDSS in the natural history studies and PDDS in NARCOMS — can only be regarded as an approximation). Mean time to obligate cane ("late cane," or PDDS = 5) in NARCOMS was approximately 30 years, which is similar to time to obligate cane $($ EDSS $=6)$ in 2 recent Canadian studies. ${ }^{1}$ The proportion of patients with a "benign" disability score (PDDS $\leq 1)$ after 20 years of disease was $18 \%$ in NARCOMS, which is nearly identical to "benign MS" prevalence in 2 population-based studies that used EDSS $=2$ as a cutoff of benignity. ${ }^{16,17}$ Notably, the proportion of benign patients in NARCOMS continued to decline after the second decade and reached its nadir of $8 \%$ after 45 years of disease. Continuous attrition of the "benign MS" group throughout the disease course was also observed in a 50-year follow-up of an incidence cohort from Gothenburg, Sweden, ${ }^{17}$ and in studies from France, ${ }^{18}$ Ireland, ${ }^{19}$ and Canada. ${ }^{20}$

We also carried out a more detailed comparison of distributions of mean ranks of PDDS (P-MSSS) in NARCOMS and mean ranks of EDSS (MSSS) in the Genetic Analysis of Multiple Sclerosis in Europeans (GAMES) consortium of European MS clinics, which served as the reference dataset for the MSSS Table. ${ }^{3}$ Overall patterns of disability accumulation were very similar in the NARCOMS and GAMES datasets early on in the disease, but NARCOMS patients tended to have a milder disability profile compared to GAMES patients with increasing disease duration. ${ }^{21}$ Milder disability of NARCOMS patients relative to GAMES patients later on in the disease may be a result of several factors: intrinsic changes in rate of disability accumulation in the mostly treated NARCOMS population, compared to untreated GAMES population; genetic difference between North American and European patients; and decreased enrollment of the most severely affected patients with longer disease duration in the NARCOMS compared to the GAMES dataset, creating mean rank inflation.

Similarities between NARCOMS and populationand clinic-based registries ${ }^{21-24}$ with respect to disability measures (time-to-cane, proportions of benign patients at 20-year mark, distribution of mean ranks of disability scores) and demographic characteristics (sex ratio, age at disease onset, employment rates) provide a measure of confidence that, notwithstanding selection bias inherent to a volunteer registry, the NARCOMS cohort is reasonably representative of the contemporary MS population at large, except, possibly, for the patients in the extreme end of the disability spectrum. The unexpectedly small proportion of bed-bound patients (PDDS $=8)$ in the DET suggests that the older and more severely impaired patients are unlikely to selfenroll into NARCOMS. We surmise that the DET more accurately reflects disability outcomes among patients in the mild-to-moderate disability range, and may not be as useful for the most disabled patients. Another limitation of our study, which applies equally to population-based natural history studies, is that the scale employed to measure disability does not assess degree of cognitive impairment. Thus, the DET reflects only the physical disability outcomes in MS. Analyses of prevalence of self-rated impairment in 11 neurologic domains as a function of disease duration in NARCOMS registrants is presented elsewhere. ${ }^{29}$

While maximum ranks of PDDS, displayed in the DET, can help patients and clinicians to better understand disease prognosis, mean ranks (P-MSSS) are more appropriate as an outcome measure in trials and observational studies. For statistical purposes, mean ranks are superior to maximum ranks (cumulative frequency) because, unlike maximum ranks, mean ranks are standardized for differences in the disease duration profiles of the subpopulations. As a result, the average of the mean ranks for all the patients in a subpopulation can be directly compared to the average of the mean ranks for all the patients in another subpopulation. In contrast, without proper adjustment, comparisons of average maximum ranks between subpopulations are confounded 
by differences in the distribution of patients among the corresponding disease duration categories of the subpopulations. This is in direct analogy with age-adjusted mortality rates where averages of the age-specific mortality rates in 2 populations are comparable only if the average in each population is weighted by the proportion of persons in each age category in the population. ${ }^{25}$ P-MSSS may be used in epidemiologic studies in much the same way as MSSS: for comparing disability profiles in 2 different large databases ${ }^{21}$ and study of calendar time trends in rates of disability accumulation. We have previously shown that MSSS scores in the New York State MS Consortium ${ }^{6}$ and the MSBase ${ }^{26}$ trend down throughout the enrollment period. A similar analysis using P-MSSS and the NARCOMS dataset is underway.

The DET represents a uniquely detailed overview of disability outcomes in a large MS cohort over several decades of disease. For patients with MS, the DET affords an opportunity to determine how their disability compares to others with similar disease duration and so provides context for more informed treatment decisionmaking, which is becoming increasingly complex with expansion of the therapeutic armamentarium in MS. For the clinical researcher, P-MSSS may prove useful as a practical and cost-effective outcome measure for comparing disability across different populations, and tracking disease progression and response to treatments in patient cohorts.

\section{AUTHOR CONTRIBUTIONS}

Ilya Kister: study concept and design, analysis and interpretation of data, drafting of the manuscript, critical revision of the manuscript for important intellectual content. Eric Chamot: study concept and design, analysis and interpretation of data, drafting of the manuscript, critical revision of the manuscript for important intellectual content, study supervision. Amber Salter: analysis and interpretation of data, drafting of the manuscript, critical revision of the manuscript for important intellectual content. Gary Cutter: study concept and design, analysis and interpretation of data, drafting of the manuscript, critical revision of the manuscript for important intellectual content, study supervision. Tamar E. Bacon: analysis and interpretation of data, drafting of the manuscript, critical revision of the manuscript for important intellectual content. Joseph Herbert: study concept and design, analysis and interpretation of data, drafting of the manuscript, critical revision of the manuscript for important intellectual content, study supervision.

\section{ACKNOWLEDGMENT}

The authors thank their colleagues, Prof. Albert Goodgold and Drs. Jonathan Howard and Lana Zhovtis-Ryerson, for their comments on the manuscript.

\section{STUDY FUNDING}

Supported by a grant from the National Multiple Sclerosis Society (RG 4542A-2) and by an award from Biogen-Idec. NARCOMS Registry is supported in part by the Consortium of Multiple Sclerosis Centers and its Foundation.

\section{DISCLOSURE}

I. Kister received research support from the National Multiple Sclerosis Society (NMSS), Jackson-Guthy Charitable Foundation, EMD-Serono/Pfizer, Inc., and Biogen-Idec, Inc. E. Chamot has research support from NIH, NMSS, and UAB Comprehensive Cancer Center-AIDS Research Center. A.R. Salter reports no disclosures. G. Cutter has served on scientific advisory boards for or received funding for travel from Millennium Pharmaceuticals, Inc., Klein Buendel, Inc., Alexion Pharmaceuticals, Inc., Androclus Therapeutics, Inc.
University of Illinois, Amgen, New York University, and Somnus Therapeutics, Inc.; receives royalties from publishing Evaluation of Health Promotion and Disease Prevention (The McGraw Hill Companies, 1984); has received honoraria from GlaxoSmithKline, Biogen Idec, Novartis, Advanced Health Media Inc., Biogen Idec, EMD Serono Inc., EDJ Associates, Inc., the National Heart, Lung, and Blood Institute, National Institute of Neurological Diseases and Stroke, National Marrow Donor Program, and Consortium of Multiple Sclerosis Centers; serves as a consultant to Peptimmune Inc., Aegis Creative Marketing, Novartis, National Industrial Sand Association, Bayer Pharmaceuticals, and Teva Pharmaceuticals Industries Ltd.; has served on independent data and safety monitoring committees for Antisense Therapeutics Limited, Sanofi-Aventis, Bayhill Pharmaceuticals, BioMS Medical Corp, DaiichiSankyo Co. Inc., GlaxoSmithKline, Genmab, Medivation Inc., PTC Therapeutics Inc., Teva Pharmaceutical Industries Ltd., Vivus Inc., NHLBI, NINDS, and NMSS; has received research support from ApopLogic Pharmaceuticals, LLC; receives research support from the NIH [NINDS 5U01NS042685-02 (PI), NINDS U01 NS45719-01A1 (PI, Coord Center)], NIAID Contract No HHSN266200400068C [Co-I], NHLBI 5R01 HL06991-02 [PI, Coord Center], NIAID N01AI30025 [Director, Coord Center], NIDR 3R01DE016684-03S109 [Co-I], NHLBI 5P50HL084923030001 [Director, Coord Center], NIDDK 1R01DK078826 [Co-I], NIAID P30AI27767 [Co-Director, Biostat Core], NIDDK 1P30DK079337 [Director, Biostat Core], and from the Consortium of Multiple Sclerosis Centers [Director NARCOMS Data Center] and the National Multiple Sclerosis Society; and serves as President of Pythagoras, Inc. T.E. Bacon reports no disclosures. J. Herbert has received personal compensation for activities with Biogen Idec, EMD Serono, Teva Neuroscience, and Bayer as a consultant. Dr. Herbert has received research support from Teva Neuroscience, Novartis, Biogen Idec, Bayer, EMD-Serono/Pfizer, BioMS and INC Research. Go to Neurology.org for full disclosures.

\section{APPENDIX}

Patient-determined disease steps (this non-copyrighted scale is an expanded version of Disease Steps introduced by Hohol et al. ${ }^{27,28}$ )

0 Normal: I may have some mild symptoms, mostly sensory, due to MS, but they do not limit my activity. If I do have an attack, I return to normal when the attack has passed.

1 Mild disability: I have some noticeable symptoms from my MS, but they are minor and have only a small effect on my lifestyle.

2 Moderate disability: I don't have any limitations in my walking ability. However, I do have significant problems due to MS that limit daily activities in other ways.

3 Gait disability: MS does interfere with my activities, especially my walking. I can work a full day, but athletic or physically demanding activities are more difficult than they used to be. I usually don't need a cane or other assistance to walk, but I might need some assistance during an attack.

4 Early cane: I use a cane or a single crutch or some other form of support (such as touching a wall or leaning on someone's arm) for walking all the time or part of the time, especially when walking outside. I think I can walk 25 feet in 20 seconds without a cane or crutch. I always need some assistance (cane or crutch) if I want to walk as far as 3 blocks.

5 Late cane: To be able to walk 25 feet, I have to have a cane, crutch, or someone to hold onto. I can get around the house or other buildings by holding onto furniture or touching the walls for support. I may use a scooter or wheelchair if I want to go greater distances.

6 Bilateral support: To be able to walk as far as 25 feet I must have 2 canes or crutches or a walker. I may use a scooter or wheelchair for longer distances.

7 Wheelchair/scooter: My main form of mobility is a wheelchair. I may be able to stand and/or take 1 or 2 steps, but I can't walk 25 feet, even with crutches or a walker.

8 Bedridden: Unable to sit in a wheelchair for more than 1 hour.

Received August 17, 2012. Accepted in final form November 26, 2012.

\section{REFERENCES}

1. Tremlett H, Zhao Y, Rieckmann P, Hutchinson M. New perspectives in the natural history of multiple sclerosis. Neurology 2010;74:2004-2015. 
2. Degenhardt A, Ramagopalan SV, Scalfari A, Ebers GC Clinical prognostic factors in multiple sclerosis: a natural history review. Nat Rev Neurol 2009;5:672-682.

3. Roxburgh RHSR, Seaman SR, Masterman T, et al. Multiple Sclerosis Severity Score: using disability and disease duration to rate disease severity. Neurology 2005;64:1144-1151.

4. International Multiple Sclerosis Genetics Consortium. Genome-wide association study of severity in multiple sclerosis. Genes Immun 2011;12:615-625.

5. Kister I, Chamot E, Bacon JH, et al. Rapid disease course in African Americans with multiple sclerosis. Neurology 2010;75:217-223.

6. Kister I, Chamot E, Bacon JH, Cutter G, Herbert J. Trend for decreasing Multiple Sclerosis Severity Scores (MSSS) with increasing calendar year of enrollment into the New York State Multiple Sclerosis Consortium. Mult Scler 2011;17: 725-733.

7. Holmén C, Piehl F, Hillert J, et al. A Swedish national postmarketing surveillance study of natalizumab treatment in multiple sclerosis. Mult Scler 2011;17:708-719.

8. NARCOMS: Multiple Sclerosis patient registry. Available at: http://www.narcoms.org. Accessed August 7, 2012.

9. Vollmer TL, Ni W, Stanton S, Hadjimichael O. The NARCOMS patient registry: a resource for investigators. Int J MS Care 1999;1:12-15.

10. Marrie RA, Cutter G, Tyry T, Campagnolo D, Vollmer T. Validation of the NARCOMS registry: diagnosis. Mult Scler 2007;13:770-775.

11. Schwartz CE, Vollmer T, Lee H. Reliability and validity of two self-report measures of impairment and disability for MS: North American Research Consortium on Multiple Sclerosis Outcomes Study Group. Neurology 1999;52: 63-70.

12. Preiningerova J, Hadjimichael O, Vollmer T, Rizzo M. Validation of patient-reported disease descriptors in the NARCOMS patient registry. Int J MS Care 2003;5:126.

13. Johnson KL, Bamer AM, Fraser R. Disease and demographic characteristics associated with unemployment among working age adults with multiple sclerosis. Int J MS Care 2009; 11:137-143.

14. Jacobs LD, Wende KE, Brownscheidle CM, et al. A profile of multiple sclerosis: the New York State Multiple Sclerosis Consortium. Mult Scler 1999;5:369-376.

15. Kaye HS, Kang T, LaPlante MP. Mobility Device Use in the United States: Disability Statistics report, (14). Washington, DC: U.S. Department of Education, National Institute on Disability and Rehabilitation Research; 2000. Available at: http://dsc.ucsf.edu. Accessed October 26, 2012.
16. Pittock SJ, McClelland RL, Mayr WT, et al. Clinical implications of benign multiple sclerosis: a 20-year populationbased follow-up study. Ann Neurol 2004;56:303-306.

17. Skoog B, Runmarker B, Winblad S, Ekholm S, Andersen O. A representative cohort of patients with non-progressive multiple sclerosis at the age of normal life expectancy. Brain 2012;135:900-911.

18. Leray E, Coustans M, Le Page E, Yaouanq J, Oger J, Edan G. 'Clinically definite benign multiple sclerosis', an unwarranted conceptual hodgepodge: evidence from a 30-year observational study. Mult Scler Epub2012 Aug 2.

19. Costelloe L, Thompson A, Walsh C, Tubridy N, Hutchinson M. Long-term clinical relevance of criteria for designating multiple sclerosis as benign after 10 years of disease. J Neurol Neurosurg Psychiatry 2008;79:1245-1248.

20. Sayao AL, Devonshire V, Tremlett H. Longitudinal followup of "benign" multiple sclerosis at 20 years. Neurology 2007;68:496-500.

21. Kister I, Chamot E, Cutter G, Salter A, Bacon T, Herbert J. Similarities in disability profiles in the North American Research Committee on Multiple Sclerosis (NARCOMS) registry and Genetic Analysis of Multiple Sclerosis in Europeans (GAMES) database. Mult Scler J 2012;18:82. Abstract.

22. Weinshenker BG, Bass B, Rice GP, et al. The natural history of multiple sclerosis: a geographically based study: I: clinical course and disability. Brain 1989;112:133-146.

23. Confavreux C, Vukusic S, Moreau T, Adeleine P. Relapses and progression of disability in multiple sclerosis. $\mathrm{N}$ Engl J Med 2000;343:1430-1438.

24. Debouverie M, Pittion-Vouyovitch S, Louis S, Guillemin F. Natural history of multiple sclerosis in a population-based cohort. Eur J Neurol 2008;15:916-921.

25. Breslow NE, Day NE. Statistical Methods in Cancer Research, Volume II, The Design and Analysis of Cohort Studies. Lyon: IARC Scientific Publication, No. 82; 1987.

26. Kister I, Chamot E, Cutter G, et al. Increasing age at disability milestones among MS patients in the MSBase Registry. J Neurol Sci 2012;318:94-99.

27. Hohol MJ, Orav EJ, Weiner HL. Disease Steps in multiple sclerosis: a simple approach to evaluate disease progression. Neurology 1995;45:251-255.

28. Hohol MJ, Orav EJ, Weiner HL. Disease Steps in multiple sclerosis: a longitudinal study comparing disease steps and EDSS to evaluate disease progression. Mult Scler 1999;5: 349-354.

29. Kister I, Bacon TE, Chamot E, Salter A, Cutter GR, Kalina JT, Herbert J. Natural history of multiple sclerosis symptoms. Int J MS Care (in press).

\section{Neurology ${ }^{\circledR}$ Launches Subspecialty Alerts by E-mail!}

Customize your online journal experience by signing up for e-mail alerts related to your subspecialty or area of interest. Access this free service by visiting http://www.neurology.org/site/subscriptions/ etoc.xhtml or click on the "E-mail Alerts" link on the home page. An extensive list of subspecialties, methods, and study design choices will be available for you to choose from-allowing you priority alerts to cutting-edge research in your field! 